\title{
Staphylococcus lugdunensis endocarditis
}

\author{
N Farrag, P Lee, R Gunney, G M Viagappan
}

\begin{abstract}
A case of Staphylococcus lugdunensis endocarditis is presented with low back pain suggesting a secondary bone focus of infection. An umbilical skin lesion may have been an additional embolic phenomenon. The case highlights the aggressive nature of $S$ lugdenensis endocarditis compared with other coagulase negative staphylococci and its association with native heart valves. In addition the importance of full identification of coagulase negative staphylococci isolated from patient samples in a case of suspected $S$ lugdenensis infection is emphasised. Antibiotic treatment may be insufficient alone in the treatment of $S$ lugdenensis endocarditis and early recourse to surgical intervention and valve replacement should therefore be considered.

(Postgrad Med f 2001;77:259-260)
\end{abstract}

Keywords: Staphylococcus lugdunensis; endocarditis; coagulase negative staphylococci

Staphylococcus lugdenensis is a coagulase negative staphylococcus (CNS) that differs from other CNS in its aggressive nature. Infection with $S$ lugdenensis usually follows a more fulminant progress and has an association with native valve endocarditis. ${ }^{1}$ Its aggressive nature therefore resembles Staphylococcus aureus and it has been reported to have similar virulence factors. ${ }^{2}$ Given the severe nature of $S$ lugdenensis endocarditis, it is important that the organism is identified promptly. In addition, such an infection should be treated aggressively as with a $S$ aureus endocarditis. We present a case of $S$ lugdenensis endocarditis in an elderly women and discuss the condition in further detail.

Microbiology,

George's Hospital,

London

N Farrag

Department of Microbiology, North Middlesex Hospital, London

P Lee

Department of Radiology, St George's Hospital, London R Gunney

Department of Microbiology, University Hospital Lewisham, High

Street, Lewisham, London SE13 6LH, UK G M Viagappan

Correspondence to: Dr Viagappan

Submitted 6 July 1999 Accepted 6 September 2000

\section{Case report}

A 78 year old women presented to hospital via her general practitioner (GP) complaining of back ache with decreased mobility, decreased appetite, and feeling generally unwell. There had been a one month history of back pain with no radiation, preceded by a two week history of general malaise and feeling unwell. It was originally of acute onset and there was no obvious history of trauma. The patient had previously been mobile and living independently, but she now had to hold onto furniture in her home to get around. She had been started on non-steroidal anti-inflammatory agents to treat the pain. There was no history of leg weakness or sensory loss. She had been treated one month previously by her GP for a presumed urinary tract infection, but there were no urinary symptoms at the time of her presenting illness. The past medical history was unremarkable.
On examination the patient was afebrile, with a temperature of $37^{\circ} \mathrm{C}$. Her blood pressure was $130 / 80 \mathrm{~mm} \mathrm{Hg}$, with a pulse rate of 80 beats/min. Her jugular venous pressure was not raised and there was no evidence of splinter haemorrhages. Bilateral ankle oedema was noted extending to the knee.

On auscultation a pansystolic murmur was heard radiating to the axilla. Examination of the respiratory system was normal. On examination of the abdomen, an oozing superficial umbilical skin lesion was noted. Her back was non-tender and no gross abnormality was found on neurological examination.

\section{INVESTIGATIONS}

On admission, the patient had a low sodium of $123 \mathrm{mmol} / 1$ (normal range 135-145) and a low potassium of $2.6 \mathrm{mmol} / \mathrm{l}(3.5-4.7)$. Her urea concentration was $6.1 \mathrm{mmol} / \mathrm{l}(2.5-8.0)$ and the creatinine was $79 \mu \mathrm{mol} / 1$ (60-110). Her liver function tests showed impairment, with a bilirubin on admission of $27 \mu \mathrm{mol} / 1(0-17)$, alanine transaminase $74 \mathrm{U} / 1$ (5-40), and an alkaline phosphatase of $187 \mathrm{U} / 1$ (50-140).

She had a normochromic, normocytic anaemia on admission with a haemoglobin of $96 \mathrm{~g} / \mathrm{l}$, a raised white cell count of $17.7 \times 10^{9} / 1$, with a neutrophil count of $15.2 \times 10^{9} / 1$. Her erythrocyte sedimentation rate was $33 \mathrm{~mm}$ in the first hour.

Chest radiography showed bilateral small pleural effusions, with possible consolidation in the left base. Lumbar spine radiography showed a grade 1 spondylolisthesis at L4 on L5. Disc space narrowing was present at all levels of the lumbar spine, but most marked at the L5/S1 level, where there was irregularity of the vertebral end plate. This raised the possibility of infection. In view of the history and the presence of anaemia and a pansystolic murmur, repeat blood cultures were taken and an echocardiogram was booked.

A staphylococcus was isolated from blood cultures and the patient was started on intravenous flucloxacillin $2 \mathrm{~g}$ every four hours, oral fusidic acid $500 \mathrm{mg}$ three times a day, and intravenous gentamicin $120 \mathrm{mg}$ three times a day. In view of the history of associated back pain with suspected endocarditis, the gentamicin was stopped after three doses and treatment continued with the flucloxacillin and fusidic acid for treatment of suspected staphylococcal endocarditis, together with possible osteomyelitis.

\section{MICROBIOLOGY RESULTS}

A staphylococcus was isolated from 11 out of 12 blood culture bottles (six sets). The staphylococcus was staphylase positive, but tube coagulase negative (Stoke's method). The staphylococcus was subsequently identified as a Slugdunensis (API Basingstoke). 


\section{Learning points}

Staphylococcus lugdenensis:

- Resembles Staphylococcus aureus in its aggressive nature.

- Has an association with native valve endocarditis.

- Reported to have similar virulence factors to $S$ aureus.

- Antibiotic treatment alone may be insufficient treatment of $S$ lugdenensis endocarditis.

- Early recourse to surgery and valve replacement should therefore be considered.

On disc sensitivity testing (Stoke's method), the isolate was sensitive to penicillin, erythromycin, methicillin, fusidic acid, gentamicin, rifampicin, and vancomycin. The minimum inhibitory concentration (MIC) and minimum bactericidal concentration (MBC) for the organism were as follows: penicillin MIC and $\mathrm{MBC}<0.03$, clindamycin MIC and MBC $<0.12$, gentamicin MIC and MBC 1 , and vancomycin MIC and MBC 1 (Sensititre UK).

A swab from the umbilical lesion grew a CNS. The patient subsequently died and a vegetation seen at postmortem examination was sent for culture and grew a CNS identified as $S$ lugdunensis (API Basingstoke).

PROGRESS ON WARD

The patient remained stable on treatment with flucloxacillin and fusidic acid for suspected $S$ lugdunensis endocarditis. She remained clinically stable on the ward until eight days into the admission, when she became drowsy and confused. On examination, her pulse rate was 72 beats/min, with a blood pressure of $80 / 40 \mathrm{~mm}$ $\mathrm{Hg}$. Her liver function tests had become raised further and fusidic acid was therefore stopped. Her urine output was noted to have declined. She was started on an intravenous infusion of normal saline. In spite of this medical intervention, the patient subsequently died.

At postmortem examination a $2 \mathrm{~cm}$ reddish, adherent, friable vegetation was seen attached to the posterior leaflet of the mitral valve from which was cultured $S$ lugdunensis. No gross abnormality was seen in the liver or spleen. There was no gross evidence of osteomyelitis. There was no evidence of cerebral thrombosis, haemorrhage, or infarction. The umbilicus showed a blackish blood tinged discharge and some crusting.

\section{Discussion}

There has been much interest in $S$ lugdenensis as a potentially aggressive pathogen. CNS are generally reported as the single most common organism causing prosthetic valve endocarditis but they are much less commonly associated with native valve endocarditis. ${ }^{1}$ The progression of endocarditis caused by CNS is usually fairly indolent.

$S$ lugdenensis differs from other CNS in its aggressive nature. It follows a more fulminant progress and has an association with native valve endocarditis. Its aggressive nature therefore resembles $S$ aureus. ${ }^{12}$ S lugdenensis appears to share certain pathogenic features to $S$ aureus. The expression of fibrinogen binding has been suggested as an important virulence factor in $S$ aureus. $S$ lugdenensis is also able to bind to this protein in a similar way. ${ }^{2}$ Other authors have also noted the aggressive nature of $S$ lugdenensis. Vandenesch et al cite three key feature of $S$ lugdenensis endocarditis compared with endocarditis caused by other CNS: a shorter, more aggressive clinical history, abscess formation around the valve, and a high mortality rate. ${ }^{3}$ Koh et al also highlighted the high mortality rate associated with S lugdenensis. Of 12 quoted cases, eight ended in fatality. ${ }^{4}$

No gross evidence of embolisation was seen internally at postmortem examination. However, the embolic nature of the organism may have been demonstrated in this case by an umbilical lesion. A swab from this lesion grew a CNS, but no further identification was performed as this was isolated before full identification of the blood culture isolate was available. A CNS isolated from a skin site is commonly considered to be part of the normal commensal flora. Given the clinical picture, this lesion may have represented an embolic phenomenon. Full identification of the umbilical isolate may have provided evidence of this. It is advisable therefore that CNS, isolated from sites where they are normally considered commensal flora, should be fully identified in a patient with suspected $S$ lugdenensis infection.

This case also illustrates the variable results that may be obtained when identifying $S$ lugdenensis in the laboratory. This feature of the organism has also been demonstrated by other authors. $^{25}$ The organism tested positive with a staphylase test, but was negative on tube coagulase testing. The final identification was performed using the API Staph system.

The patient was initially treated with intravenous flucloxacillin and gentamicin. This regimen was changed to intravenous flucloxacillin and oral fusidic acid in view of the history of low back pain, which was suggestive of a possibly bony source or bony embolic site of infection. The patient's condition stabilised on this treatment and she appeared to be doing well on medical treatment.

The case shows that $S$ lugdenensis behaves in a much more aggressive manner than other CNS. Antibiotic therapy may be insufficient alone in the treatment of $S$ lugdenensis endocarditis. Early recourse to surgery and valve replacement should therefore be considered.

\footnotetext{
1 Etienne J, Pangon B, Leport C, et al. Staphylococcus lugdunensis endocarditis. Lancet 1989;i:390.

2 Paulsson M, Petersson A, Ljungh A. Serum and tissue bindPaulsson M, Petersson A, Ljungh A. Serum and tissue bind-
ing and cell surface properties of Staphylococcus lugdunening and cell surface properties of Stap
sis. F Med Microbiol 1993;38:96-102.

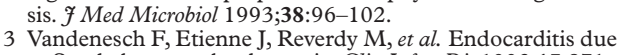
to Staphylococcus lugdunensis. Clin Infect Dis 1993;17:8716.

4 Koh TW, Brecker SJD, Layton CA. Successsful treatment of Staphylococcus lugdunensis endocarditis complicated by multiple emboli: a case report and review of the literature. Int $\mathcal{F}$ Cardiol 1996;55:193-7.

5 Barker KF, O'Driscoll JC, Bhargava A. Staphylococcus lugBarker KF, O'Driscoll JC, Bhargava A.
dunensis. $\mathcal{F}$ Clin Pathol 1991;44:873-4.
} 